\title{
Two cases of Alzheimer's disease showing deterioration of behavioral and psychological symptoms of dementia induced by switching from rivastigmine to donepezil
}

\author{
This article was published in the following Dove Press journal: \\ Neuropsychiatric Disease and Treatment \\ 27 December 2012 \\ Number of times this article has been viewed
}

\author{
Takemi Kimura \\ Junichi Takamatsu \\ Division of Clinical Research, National \\ Hospital Organization Kikuchi \\ Hospital, Koshi, Kumamoto, Japan
}

\begin{abstract}
Rivastigmine, galantamine, and memantine, in addition to donepezil, which has been on the market over 10 years, have been available for the treatment of Alzheimer's disease (AD) since 2011 in Japan, leading a new stage in the medical treatment of AD. We studied two AD patients showing sudden deterioration of behavioral and psychological symptoms of dementia (BPSD) associated with switching from rivastigmine to donepezil after the clinical trial of rivastigmine. In the patients, rivastigmine seemed to be more beneficial than donepezil for the control of BPSD. Although It was not obvious whether their different responses to the two cholinesterase inhibitors were due to the different pharmacological profiles, ie, the presence of inhibition of butyrylcholinesterase in rivastigmine, a particular cholinesterase inhibitor might be more effective in particular AD cases. Further investigations are needed to confirm the difference, and to identify the measures for selecting the most appropriate medication for each AD patient.
\end{abstract}

Keywords: rivastigmine, donepezil, cholinesterase inhibitors, switching, behavioral and psychological symptoms of dementia, neuropsychiatric inventory, Japanese, Alzheimer's disease

\section{Introduction}

In Japan, there was no choice of medication for the treatment of Alzheimer's disease (AD), other than donepezil, for more than a decade, as rivastigmine, galantamine, and memantine were not approved by the Japanese Ministry of Health, Labour and Welfare until 2011. The recent availability of the three medications is leading to a new stage in the medical treatment of AD in Japan. Donepezil and galantamine block only acetylcholinesterase, while rivastigmine is a dual inhibitor of acetylcholinesterase and butyrylcholinesterase. On the other hand, galantamine is a potent allosteric potentiating ligand of nicotinic receptors. ${ }^{1}$ Memantine blocks the N-methyl-D-aspartate receptor ${ }^{2}$ and thereby protects nerve cells from excess stimulation by glutamate. Since these drugs have different pharmacological profiles, one should select the appropriate drug carefully, according to the AD progression and symptoms of the patients. However, there are few instances of using the newly approved drugs for the treatment of AD in Japan. Here, we report on two patients with AD who showed deterioration in the behavioral and psychological symptoms of dementia (BPSD) associated with switching to donepezil from rivastigmine after a clinical Phase III trial.

The patients and their families agreed to the publication of this report in writing. Their personal details were rendered anonymous so as not to reveal their identities. 


\section{Cases}

\section{Patient I}

A 76-year-old woman without family history of AD presented with a 5-year history of cognitive decline and a decrease in her activities of daily living. She needed the help of her family for daily activities such as excretion, bathing, and changing her clothes. On the Mini-Mental State Examination (MMSE), her total score was 11 points. No particular abnormality was found upon blood and urine examination. A cranial MRI revealed moderate atrophy of the cerebral cortices, an enlargement of the inferior horn of the right lateral ventricle, and a slight ischemic change in the deep white matter. Brain bloodflow scintigraphy showed a right dominant decline in the parietotemporal lobes and a decline in the posterior cingulate gyrus. Clinically, she was slightly apathetic and had no neurological abnormalities. There was no use of drugs or physical or neurological factors to explain her cognitive disturbance. Based on these findings, she was diagnosed with $\mathrm{AD}$.

The patient participated in the clinical study of rivastigmine - a 24-week, multicenter, randomized, double-blind, placebo-controlled, parallel-group, dose-finding study evaluating the efficacy, safety, and tolerability of the oncedaily rivastigmine patch formulation in patients with probable AD (MMSE score of 10-20) ${ }^{3}$ and an accompanying, open-label, 52-week, extension study. One week after the extension study with a rivastigmine patch $(18 \mathrm{mg} /$ day $)$, she started treatment with donepezil at a dose of $3 \mathrm{mg} /$ day. She suffered from insomnia 3 days later and did not follow family advice, eg, walking around outside regardless of bad weather and ending up being protected by the police. In spite of it being a very hot day, she went out and lost her way, and was subsequently put under protective custody at a police station. She did not have these BPSD symptoms prior to or during rivastigmine treatment. Consequent to these occurrences, she was admitted to National Hospital Organization, Kikuchi Hospital. Her scores on the Neuropsychiatric Inventory (NPI) before taking donepezil and at admission were 17 and 38 points, respectively (Figure 1A). Her agitation, irritability, and loitering tendencies improved after her discontinuation of donepezil and subsequent to her being prescribed $5 \mathrm{~g}$ /day Yokukansan (TJ-54; Tsumura Co, Tokyo, Japan) within a week.

Yokukansan (in Japan) is a traditional Asian herbal medicine that has been approved for insomnia and anxiety disorders by the Japanese Ministry of Health, Labour and Welfare; prescriptions for it are covered under the National Health Insurance plan.

\section{Patient 2}

A 56-year-old woman who had no family history of AD showed a 5-year history of cognitive decline. Impairment in cooking, washing, changing clothes, and temporal orientation were evident. Her total MMSE score was 12 points. There were no abnormalities found in hematological and blood chemistry examinations and urinalysis. Her cranial MRI images showed moderate atrophy of the parietal lobes, mild enlargement of the lateral ventricle, and no ischemic changes. Upon clinical examination, there was apathy, but no neurological abnormalities were found. We found no drug-related or physical or neurological factors to have caused her cognitive disturbance. From these findings, she was diagnosed with AD.

The patient took part in the clinical rivastigmine study. Two weeks after the extension study with a rivastigmine patch (18 mg/day), she was prescribed donepezil ( $3 \mathrm{mg} /$ day) according to the wishes of her husband. She began to go out early in the morning 4 days after starting donepezil; she loitered all day and became agitated and aggressive toward family members 6 days later. Consequently, her husband urgently took her to our hospital. Her NPI scores before taking donepezil and when undergoing NPI were 20 and 40 points, respectively (Figure 1B). Her loitering, agitation, and aggression decreased after her discontinuation of donepezil and subsequent to her being prescribed tandospirone (30 $\mathrm{mg} /$ day) and Yokukansan (7.5 g/day) within a week.

Tandospirone is an anxiolytic and antidepressant used in China and Japan. It is a member of the azapirone and piperazine chemical classes and is closely related to other agents such as buspirone and gepirone. Tandospirone acts as a potent and selective 5-HT1A receptor partial agonist.

\section{Discussion}

Our patients suffered from deterioration of BPSD associated with a switching from rivastigmine to donepezil. Comparing the pharmacological profiles of donepezil and rivastigmine, it is known that donepezil is a specific acetylcholinesterase inhibitor, while rivastigmine is a dual cholinesterase inhibitor (ChEI) that is capable of inhibiting butyrylcholinesterase, another enzyme involved in acetylcholine degradation. ${ }^{4}$ The different efficacies of these $\mathrm{ChEIs}$ are also reported in clinical settings: ${ }^{5-7}$ however, it is not evident that these differences are due to the different pharmacological profiles mentioned above. It is also conceivable that the pharmacological differences among the ChEIs result in different profiles of adverse events, including neuropsychiatric symptoms. The occurrence and presentations of BPSD varied between our 

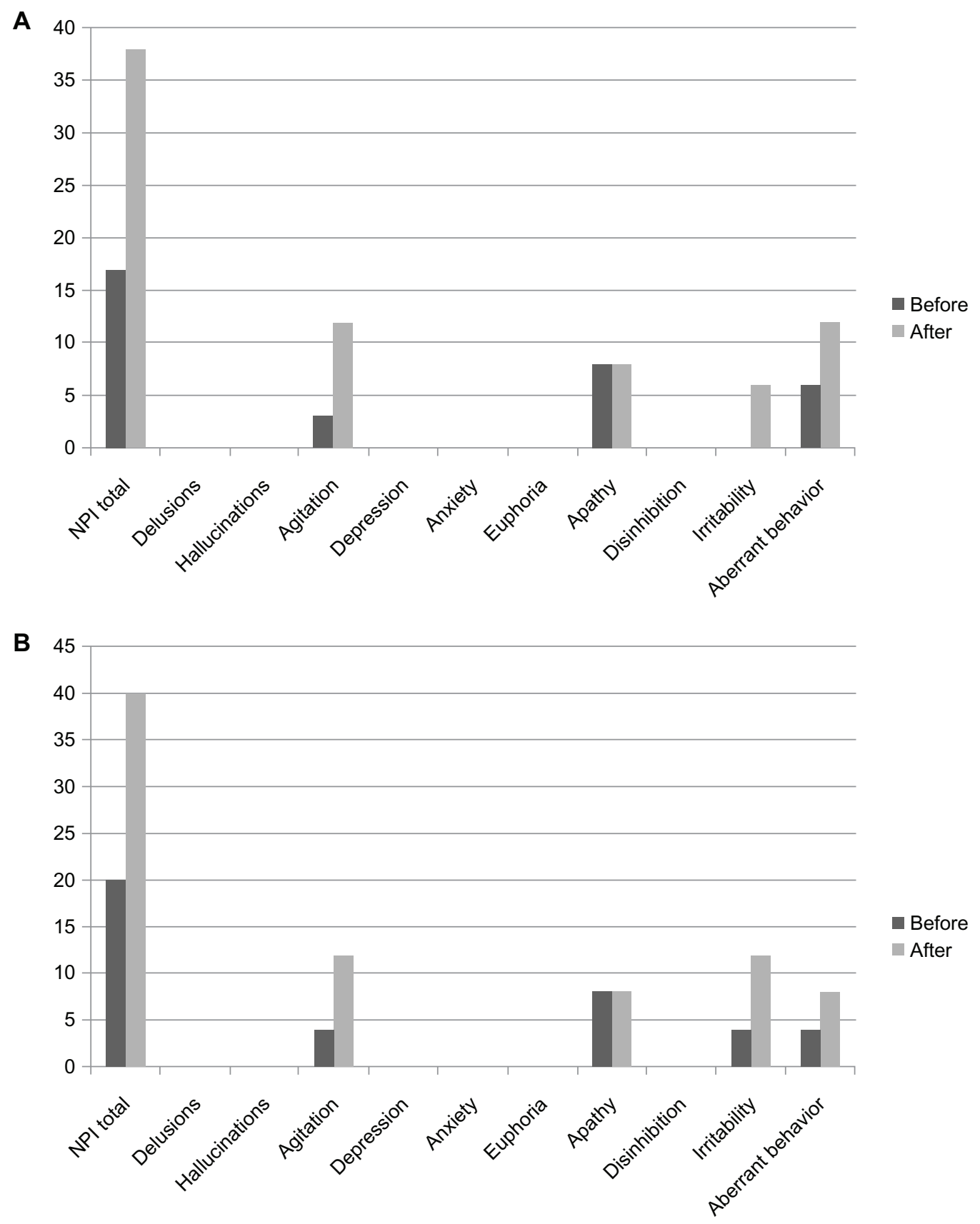

Figure I Changes in subscales of the Neuropsychiatric Inventory before and after switching from rivastigmine to donepezil in patient I (A) and patient 2 (B) results.

patients, and they could not explained by neuropathological differences between them. The manifestation of BPSD may be affected by patient profiles, including personality and environmental factors such as caregiving. In AD patients, sensitivity to psychoactive drugs may also vary, and some patients can be prone to drug-induced psychiatric events. ${ }^{8}$ It is reported that some $\mathrm{AD}$ patients show hostility or agitation accompanying their donepezil treatment, ${ }^{9-11}$ suggesting the possible exacerbation of neuropsychiatric symptoms by donepezil treatment. We occasionally experience dementia patients whose BPSD has been worsened by donepezil. ${ }^{12,13}$ It can be hypothesized that, in some patients, neuropsychiatric symptoms may be induced and enhanced by donepezil but that they are not, or are less evidently so, by rivastigmine. With this hypothesis, we should carefully evaluate the BPSD of patients after switching ChEIs, because the switch may cause BPSD to deteriorate, as it did in our patients. It also should be mentioned that we should be cautious when switching the medication in the reverse direction, ie, from donepezil to rivastigmine, as the switch may potentially 
cause other adverse effects in some patients. Besides the two drugs' different pharmacological profiles, their difference in formulation might be related to the deteriorated BPSD in the observed cases.

Rivastigmine is applied as a patch formulation, while donepezil is an oral-delivery formulation. With the patch, the pharmacologically active ingredient is continuously absorbed through the skin barrier composed of the epidermis and dermis, resulting in an unfluctuating blood concentration of the compound throughout the treatment. ${ }^{14}$ The pharmacokinetic profile of donepezil is known to be preferable, not least due to its long plasma half-life (t1/2) resulting in a considerably high trough level with oral applications at 24-hour intervals. However, like other oral medications, peak plasma concentration $\left(\mathrm{C}_{\max }\right)$ after oral administration is unavoidable. In this regard, the deterioration in behavioral and psychological symptoms observed in the two clinical cases have been caused by their different pharmacokinetic profiles. The high peak-blood concentration of ChEI or the temporal fluctuation of the blood concentration may have caused such adverse symptoms. It may also be possible that both pharmacological and pharmacokinetic differences were involved in the cases we observed. Nevertheless, the cases should be considered, in the context of pharmacology and pharmacokinetics, to have been exceptional ones that showed patients with profiles prone to negative effects from donepezil treatment.

A randomized, double-blind clinical trial comparing the efficacy and safety of rivastigmine and donepezil ${ }^{5}$ showed no significant difference in overall efficacy as determined by the Severe Impairment Battery. ${ }^{15}$ A slight but significant difference was observed in the Alzheimer's Disease Cooperative Study-Activities of Daily Living Inventory, ${ }^{16}$ the assessment battery on activities of daily living, suggesting pharmacological difference between the two medications. ${ }^{5}$ In the study, gastrointestinally adverse events were more frequent in the patients treated with rivastigmine, because it was given in capsule instead of patch formulation. There was no difference in the frequency of neuropsychiatric events in the study. As different responses to different medications may have occurred only in some patients, it is not possible to detect such responses in large clinical trials involving hundreds of patients. The discovery of biomarkers to discriminate patients with symptoms exacerbated by participation, together with careful and intensive observation of patients, will be needed to generalize our observations in this report.

We have reported on two AD patients who showed deterioration of BPSD induced by switching from rivastigmine to donepezil. Our observations might give general practitioners information useful for noticing possible unfavorable reactions caused by changing medications from the same therapeutic class. Further collection and verification of cases is required to find patient populations with the optimum medications and/or risk factors, in order to determine the most appropriate medications to administer, according to the symptoms, disease stages, or putative subtypes of the disease.

\section{Acknowledgments}

Dr Kimura reviewed the medical chart, provided data interpretation, and edited the manuscript. Dr Takamatsu provided data interpretation and scientific writing for publication. The authors thank the patients and their relatives for their cooperation. Written, informed consent was obtained from the patients and their families for the publication of this case report.

\section{Disclosure}

The authors report no conflicts of interest in this work.

\section{References}

1. Villarroya M, Garcia AG, Marco JL. New classes of AChE inhibitors with additional pharmacological effects of interest for the treatment of Alzheimer's disease. Curr Pharm Des. 2004;10(25):3177-3184.

2. Akaike A, Kume T. Neurodegenerative disease and nicotinic receptor. J Clin Exp Med. 2004;210(7-8):687-690. Japanese.

3. Nakamura Y, Imai Y, Shigeta M, et al. A 24-week, randomized, double-blind, placebo-controlled study to evaluate the efficacy, safety and tolerability of the rivastigmine patch in Japanese patients with Alzheimer's disease. Dement Geriatr Cogn Dis Extra. 2011;1(1): 163-179.

4. Weinstock M. Selectivity of cholinesterase inhibition: clinical implications for the treatment of Alzheimer's disease. CNS Drugs. 1999;12(4):307-323.

5. Bullock R, Touchon J, Bergman H, et al. Rivastigmine and donepezil treatment in moderate to moderately-severe Alzheimer's disease over a 2-year period. Curr Med Res Opin. 2005;21(8):1317-1327.

6. Bullock R, Bergman H, Touchon J, et al. Effect of age on response to rivastigmine or donepezil in patients with Alzheimer's disease. Curr Med Res Opin. 2006;22(3):483-494.

7. Blesa R, Bullock R, He Y, et al. Effect of butyrylcholinesterase genotype on the response to rivastigmine or donepezil in younger patients with Alzheimer's disease. Pharmacogenet Genomics. 2006;16(11):771-774.

8. Cumbo E. Differential effects of rivastigmine, galantamine and donepezil on behavioral and psychological symptoms in patients with Alzheimer's disease: 18-month, randomized, open-label trial. Prim Care Comm Psych. 2005;10(3):95-102.

9. Winblad B, Engedal K, Soininen H, et al. A 1-year, randomized, placebocontrolled study of donepezil in patients with mild to moderate AD. Neurology. 2001;57(3):489-495.

10. Bouman WP, Pinner G. Violent behavior-associated with donepezil. Am J Psychiatry. 1998;155(11):1626-1627.

11. Bianchetti A, Trabucchi M, Cipriani G. Aggressive behaviour associated with donepezil treatment: a case report. Int J Geriatr Psychiatry. 2003;18(7):657-658. 
12. Kimura T, Takamatsu J. Pilot study of pharmacological treatment for frontotemporal dementia: risk of donepezil treatment of behavioral and psychological symptoms. Geriatr Gerontol Int. In press 2013.

13. Kimura T, Hayashida H, Miyauchi D, Takamatsu J. Drug-resistant dementia with Lewy bodies: case report of 3 patients showing good response to aripiprazole treatment. Clin Psychiatry. 2010;52(6):575-582. Japanese.

14. Mercier F, Lefèvre G, Huang HL, Schmidli H, Amzal B, AppelDingemanse $S$. Rivastigmine exposure provided by a transdermal patch versus capsules. Curr Med Res Opin. 2007;23(12):3199-3204.
15. Panisset M, Roudier M, Saxton J, Boller F. Severe impairment battery. A neuropsychological test for severely demented patients. Arch Neurol. 1994;51(1):41-45.

16. Galasko D, Bennett D, Sano M, Marson D, Kaye J, Edland SD; Alzheimer's Disease Cooperative Study. ADCS Prevention Instrument Project: assessment of instrumental activities of daily living for community-dwelling elderly individuals in dementia prevention clinical trials. Alzheimer Dis Assoc Disord. 2006;20 Suppl 3:S152-S169.

\section{Publish your work in this journal}

Neuropsychiatric Disease and Treatment is an international, peerreviewed journal of clinical therapeutics and pharmacology focusing on concise rapid reporting of clinical or pre-clinical studies on a range of neuropsychiatric and neurological disorders. This journa is indexed on PubMed Central, the 'PsycINFO' database and CAS
The manuscript management system is completely online and includes a very quick and fair peer-review system, which is all easy to use. Visit http://www.dovepress.com/testimonials.php to read real quotes from published authors.

Submit your manuscript here: http://www.dovepress.com/neuropsychiatric-disease-and-treatment-journal 